\title{
Putting Machine Vision and Machine Learning at Work in Human Monitoring Applications
}

\author{
Robert Laganière \\ Faculty of Engineering, University of Ottawa \\ Canada
}

\begin{abstract}
In the recent years, deep learning and convolutional networks have revolutionized the research in computer vision leading to impressive performance improvements. While initially focusing on image classification problems, deep learning can nowadays perform accurate object detection, a fundamental and essential task in many computer vision problems. For the detection of humans and human features, many deep architectures have been proposed. Approaches based on region proposals are often considered the most accurate but might have difficulties detecting smaller objects. The Single-Shot detection architecture constitutes another interesting alternative from which real-time detectors running on powerconstrained devices can be designed. This talk will present some object detection architectures and present recent results for the problems of customer tracking in retail stores and for pedestrian and driver detection in autonomous driving.
\end{abstract}

